\title{
Acknowledgement to Reviewers of International Journal of Turbomachinery, Propulsion and Power (IJTPP) in 2018
}

\author{
IJTPP Editorial Office \\ MDPI, St. Alban-Anlage 66, 4052 Basel, Switzerland \\ Published: 9 January 2019
}

Rigorous peer-review is the corner-stone of high-quality academic publishing. The editorial team greatly appreciates the reviewers who contributed their knowledge and expertise to the journal's editorial process over the past 12 months. In 2018, a total of 19 papers were published in the journal, with a median time to first decision of 53 days and a median time to publication of 91 days. The editors would like to express their sincere gratitude to the following reviewers for their cooperation and dedication in 2018:

\begin{tabular}{|c|c|}
\hline Ameduri, Salvatore & Hirsch, Charles \\
\hline Arts, Tony & Hupfer, Andreas \\
\hline Barigozzi, Giovanna & Ivanov, Mikhail \\
\hline Bauinger, Sabine & Kerres, Bertrand \\
\hline Bellucci, Juri & Krewinkel, Robert \\
\hline Biesinger, Thomas & Lepot, Ingrid \\
\hline Cadrecha, David & Lipatnikov, Andrei \\
\hline Cagnone, Jean-Sébastien & Lutum, Ewald \\
\hline Caignaert, Guy & Mathioudakis, Kostas \\
\hline Cantrak, Djordje & Meinke, Matthias \\
\hline Cattanei, Andrea & Muntean, Sebastian \\
\hline Chew, John & Nedeljkovic, Milos \\
\hline Coull, John & Ohta, Yutaka \\
\hline Cox, Graham & Ott, Peter \\
\hline Cravero, Carlo & Pacciani, Roberto \\
\hline Dazin, Antoine & Pavesi, Giorgio \\
\hline De Palma, Pietro & Poncet, Sébastien \\
\hline Deckers, Mathias & Porreca, Luca \\
\hline Dohmen, Hans Josef & Qiaorui, Si \\
\hline Dumas, Michel & Salvadori, Simone \\
\hline Flaszynski, Pawel & Sandham, Neil D \\
\hline Fogla, Navin & Schnös, Markus \\
\hline Fox, Michael D. & Scrivener, Colin \\
\hline Frey, Christian & Sedlar, Milan \\
\hline Funazaki, Ken-ichi & Shahpar, Shahrokh \\
\hline Godard, Antoine & Sieverding, Claus \\
\hline Gourdain, Nicolas & Uruba, Václav \\
\hline Grönman, Aki & Vázquez-Díaz, Raúl \\
\hline Harley, Peter & Vera-Morales, Maria \\
\hline Hiernaux, Stéphane & Verstraete, Tom \\
\hline Hiller, Sven-Jürgen & Wiedermann, Alexande \\
\hline
\end{tabular}

(C) 2019 by the authors. Licensee MDPI, Basel, Switzerland. This article is an open access article distributed under the terms and conditions of the Creative Commons Attribution (CC BY) license (http://creativecommons.org/licenses/by/4.0 